\title{
Fungi causing powdery mildew on plants of a Botanical Garden in Southern Finland
}

\section{Ville J. Heiskanen ${ }^{1,2,}$, Jari P.T. Valkonen ${ }^{1}$}

1 Department of Agricultural Sciences, University of Helsinki, Latokartanonkaari 7, Fl-00014 Helsinki, Finland

2 Kumpula Botanic Garden, Jyrängöntie 2, Fl-00014 Helsinki, Finland

${ }^{*}$ Corresponding author:

villejuhaniheiskanen@gmail.com

Keywords: botanic garden, Erysiphales, internal transcribed spacer (ITS), powdery mildew,

Southern Finland

Abbreviations: PMCF, Powdery Mildew-Causing Fungi

Article info:

Received: 11 November 2020

Accepted: 05 April 2021

Published online: 07 June 2021

Corresponding Editor: Risto Kasanen

\section{Abstract}

Fungi that cause powdery mildew on plants are plant pathogenic parasites (Erysiphales) and can significantly reduce the ornamental value of plants and cause significant yield losses among cultivated plants. In this study, 94 plant accessions infected with powdery mildew were observed in Kumpula Botanic Garden, Helsinki, Finland, in 2015. The taxonomic affiliation and species richness of powdery mildew fungi were investigated. Morphological studies by microscope distinguished only 14 fungal species, whereas further comparisons of internal transcribed spacer (ITS) sequences enabled the identification of 28 species. Hence, ITS sequencing improved the reliability of species determination, as compared with the use of morphological characteristics only. The vegetation in an area of six hectares supported a wide range of fungi that cause powdery mildew as well as hyperparasitic microbes, which may balance the impact of pathogens in host plants. The findings of this study emphasize the role of botanical gardens in protecting biological diversity in urban areas. 


\section{Introduction}

The fungi in the order Erysiphales are obligate parasites that cause symptoms of powdery mildew and crop losses in a wide range of cultivated agricultural and horticultural crops, including ornamental plants (Glawe 2008). Globally, ca. 900 powdery mildew-causing fungi (PMCF) have been described (Braun \& Cook 2012). However, diversity among PMCF in the Northern Hemisphere is more limited due to the short growing season and early death of host plants due to frost. In fact, diversity of PMCF in the Northern Hemisphere hasn't been studied extensively. Botanical gardens in the Northern Hemisphere offer the possibility for studying the diversity of PMCF in this region, as they contain a wide range of plant species that are adapted to a northern climate. Such studies are relatively rare and have so far focused mainly on single taxa rather than on the analysis of overall diversity of PMCF within a certain area (Korytnanskaya 2010; Mieslerová et al. 2020a; Mieslerová et al. 2020b).

Over 100 PMCF have been identified and are listed in the Finnish Biodiversity Information Facility (Salo et al. 2019), and 256 natural host-fungus combinations associated with powdery mildew have been documented in Finland (Weltzien 1978). Kumpula Botanic Garden of University of Helsinki (subsequently referred to as KBG) hosts a collection of ca. 1500 plant taxa, consisting of plants that have been introduced from different areas of the Northern Hemisphere with bioclimatic habitats resembling Southern Finland (Schulman \& Hällfors 2012). Powdery mildew is common and can significantly reduce the ornamental value of plants in the garden. The large and diverse collection of plant species in the garden may host a wide range of non-native PMCF from other countries.

PMCF can be cumbersome to study because hyphae may not be long-lasting, PMCF cannot grow on artificial media and need a living host to survive, and the characteristic structures used for identification by microscopy may be difficult to find and/or maintain. These obstacles can be alleviated by use of DNA-based methods, such as analysis of the internal transcribed spacer (ITS) sequences between the small and large subunit of ribosomal RNA genes. ITS sequences are used as universal barcodes for fungi
(Schoch 2012) allowing identification of species based on their ITS sequences and comparisons with those deposited in gene banks (Benson et al. 2013).

The aim of this study was to use morphological characteristics and molecular techniques, such as barcodes based on ITS sequences, to determine the PMCF species infecting plants in KBG, to study species richness of PMCF, and to find out whether the population of PMCF differs from the species previously characterized in Finland.

\section{Materials and methods}

\author{
Collection of samples for analysis of \\ morphology and ITS sequences
}

KBG is located at latitude $60.192059(\mathrm{~N})$ and longitude 24.945831(E) in Southern Finland. In total, 94 plant accessions with symptoms of PMCF (Fig. 1) were observed during the growth season of 2015 (May 18th to September 30th), and 70 herbarium samples were collected. Plant disease symptoms were surveyed once a week. Samples were taken from symptomatic parts, dried between sheets of blotting paper, and preserved for further examination (Table 1). In addition, powdery mildew (mycelia) was scraped off of 42 plants, transferred into Eppendorf tubes, and frozen at $-18{ }^{\circ} \mathrm{C}$ for DNA analysis.

\section{Morphological examination of fungi}

All dried samples from the herbarium were examined and measured with a Researcher Bino II light microscope (Bresser GmbH, Germany) that was equipped with a 3-megapixel camera and MicroCamLab software (v. 7.3.1.8). Fresh mycelia, were transferred onto a microscope slide covered with a piece of adhesive tape and placed on top of a drop of water (Heffer et al. 2006). Characteristics of the mycelium (color, width, growth habit, and shape of appressoria), anamorphs (size and shape of conidia and conidiophores; observation of conidiogenesis) and teleomorphs stages (size and color of chas- 

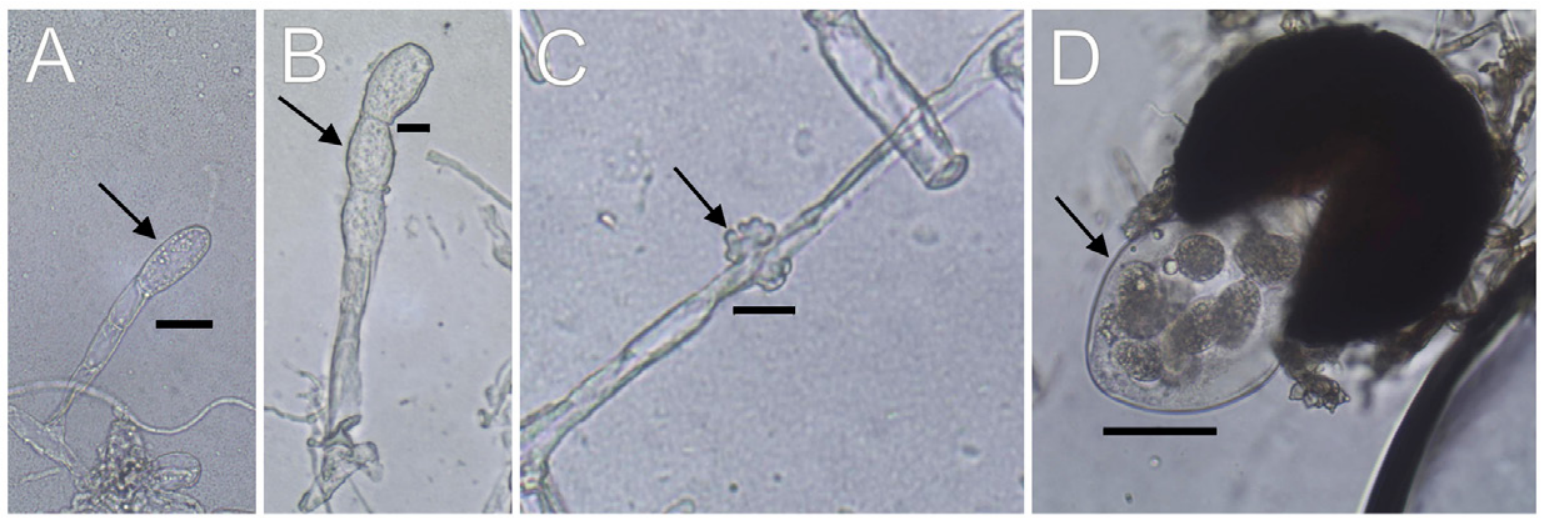

Fig. 1. Symptoms of powdery mildew on different hosts. (a) Viburnum lantana (OOXX-0153), (b) Salix caprea (2010-0939), and (c) Acer platanoides (00XX-0004). Numbers in parentheses indicate accession numbers for individual plant specimens.

mothecia; shape of appendages; shape, size, and color of asci and ascospores) were recorded. Species identification of PMCF was based on the descriptions of Braun (1995) and Braun \& Cook (2012).

\section{DNA isolation and design of PMCF-specific primers}

DNA was extracted from the fungal mycelia using DNeasy Plant Mini Kit (Qiagen GmbH, Hilden, Germany). All 42 samples were screened first by polymerase chain reaction (PCR) using the universal primers ITS5/ITS4 (White et al. 1990) to ensure that samples contained fungal DNA. Subsequently, PMCF-specific primers were designed by downloading rDNA sequences of powdery mildew-causing fungi from NCBI GenBank (Benson et al. 2013): AB022346.1, AB022347.1, AB022349.1, AB022354.1, AB022364.1, AB022365.1, AB022366, AB022369.1,АB022373.1,АB022374.1,АB022398.1, AB022399.1,AB022402.1, АB022404.1,AB022405.1, $\mathrm{AB} 022410.2, \mathrm{AB} 022411.1, \mathrm{AB} 022419.1, \mathrm{AB} 033477.1$, AB033479.1, АВ033482.1,АB077619.1,АB077671.1, AB080411.1,AB080470.1,AB103069.1,AB103078.1, $\mathrm{AB} 103370.1, \mathrm{AB} 193465.1, \mathrm{AB} 237812.1$, and AF021796.1. These sequences were aligned with MultAlin software (Corpet 1988) and used to design primers for PCR (18S-2F, 28S-4R, and 28S-6R) and sequencing (5.8S-F, 5.8S-2F, 5.8S-R, and 5.8S-3R) (Fig. 2).

\section{PCR reactions}

The whole ITS region (ITS1 + 5.8S + ITS2), which included partial $18 \mathrm{~S}$ and $28 \mathrm{~S}$ regions (800-900 bp), was amplified by PCR using the primer set $18 \mathrm{~S}-2 \mathrm{~F} / 28 \mathrm{~S}-4 \mathrm{R}$ or $18 \mathrm{~S}-2 \mathrm{~F} / 28-6 \mathrm{R}$ (Fig. 2). PCR reactions consisted of a final volume of $25 \mu \mathrm{l}$ containing $1 \mu \mathrm{l}$ DNA template, $2.5 \mu \mathrm{l}$ of Optimized 10x DyNAzyme buffer, $0.5 \mu 1$ of $10 \mathrm{mM}$ dNTPs (Thermo Fisher Scientific Baltics, UAB, Vilnius, Lithuania), $1 \mu \mathrm{l}$ of $10 \mu \mathrm{M}$ forward primer (18S-2F), $1 \mu \mathrm{l}$ of 10 $\mu \mathrm{M}$ reverse primer (28S-4R or $28 \mathrm{~S}-6 \mathrm{R})$, and $0.25 \mu \mathrm{l}$ of $2 \mathrm{U} / \mu 1$ DyNAzyme II DNA polymerase (Thermo Fisher Scientific Baltics, UAB, Vilnius, Lithuania). Thermal cycling included an initial denaturation at $94{ }^{\circ} \mathrm{C}$ for $1 \mathrm{~min}$, followed by 30 cycles of denaturation at $94{ }^{\circ} \mathrm{C}$ for $30 \mathrm{~s}$, annealing at $50{ }^{\circ} \mathrm{C}$ for $30 \mathrm{~s}$, and elongation at $72{ }^{\circ} \mathrm{C}$ for $40 \mathrm{~s}$ and a final extension of 8 min at $72{ }^{\circ} \mathrm{C}$. All PCR products were electrophoresed in an agarose gel with a $1 \%$ Tris-acetate-EDTA buffer (Sigma-Aldrich Co., St. Louis, MO, USA). Ethidium bromide was added $(2.5 \mathrm{mg} / \mathrm{ml})$ to the gel to visualize the PCR products. 
Table 1. Ninety-four host plants from KBG (Helsinki, Finland) on which symptoms of powdery mildew were observed and the methods used for sampling.

\section{Host plant a, b}

Abelmoschus esculentus

Acer negundo (H) (Amp) (9)

Acer platanoides $(\mathrm{H})$ (Amp)

Acer platanoides

Acer platanoides $(H)$ (Amp)

Acer platanoides

Acer tataricum subsp. ginnala

Acer tataricum subsp. ginnala (H) (Amp) (40)

Acer tataricum subsp. tataricum $(\mathrm{H})$

Acer tataricum subsp. tataricum (H) (Amp)

Aconitum napellus (H) (13)

Alchemilla indet.

Arctium lappa var. edule (H) (Amp) (25)

Avena sativa $(H)(37)$

Avena strigosa

Brassica oleracea var. sabellica $(H)$

Campanula bononiensis $(\mathrm{H})$

Centaurea phrygia (H) (Amp) (10)

Clematis recta $(H)(31)$

Cucumis sativus $(H)(35)$

Cucurbita maxima $(\mathrm{H})$

Cucurbita moschata $(\mathrm{H})$

Cucurbita pepo (H) (Amp)

Cucurbita pepo $(\mathrm{H})$

Cucurbita pepo (H) (Amp) (36)

Cucurbita pepo $(\mathrm{H})$

Delphinium elatum $(\mathrm{H})(38)$

Delphinium elatum $(\mathrm{H})$

Delphinium indet. $(H)(1)$

Echium maculatum $(\mathrm{H})(28)$

Echium vulgare

Euonymus europaeus $(\mathrm{H})$

Euonymus europaeus

Geranium pretense $(H)$ (39)

Geranium sanquineum $(H)(7)$

\section{Family}

Malvaceae

Sapindaceae

Sapindaceae

Sapindaceae

Sapindaceae

Sapindaceae

Sapindaceae

Sapindaceae

Sapindaceae

Sapindaceae

Ranunculaceae

Rosaceae

Asteraceae

Poaceae

Poaceae

Brassicaceae

Campanulaceae

Asteraceae

Ranunculaceae

Cucurbitaceae

Cucurbitaceae

Cucurbitaceae

Cucurbitaceae

Cucurbitaceae

Cucurbitaceae

Cucurbitaceae

Ranunculaceae

Ranunculaceae

Ranunculaceae

Boraginaceae

Boraginaceae

Celastraceae

Celastraceae

Geraniaceae

Geraniaceae
Collected c

Accession

2010-0348

31.8.

1992-0060

00XX-0004

00XX-0167

00XX-0166

00XX-0003

1994-0891

2008-0104

2005-0017

3.8

10.9

2008-0161

20.8.

1995-0451

2008-1029

2010-0296

20.8

2009-0567

20.8 .

2014-0350

2013-0024

29.9.

2012-0735

30.9 .

1992-0147

30.9 .

2004-0502

23.9.

20.8.

2013-0042

23.9 .

2015-0123

23.9.

2015-0125

23.9.

2015-0176

23.9.

2015-0175

8.9.

2015-0124

23.9

2015-0300

3.8.

2006-0690

31.8.

1995-0091

3.8.

2008-0149

3.8.

2012-0627

2011-0721

992-0099

996-0337

2001-0068

10.9.

1977-0512 
Table 1. continues on the next page

\begin{tabular}{|c|c|c|c|}
\hline Host planta, b & Family & Collected c & Accession \\
\hline Geum urbanum & Rosaceae & & $1996-0284$ \\
\hline Hieracium umbellatum $(\mathrm{H})$ & Asteraceae & 30.9 & $2010-1360$ \\
\hline Hordeum vulgare $(\mathrm{H})$ & Poaceae & 20.8. & $2009-0568$ \\
\hline Hypericum ascyron (H) (12) & Hypericaceae & 10.9 & $1993-0728$ \\
\hline Hypericum maculatum & Hypericaceae & & 2007-0699 \\
\hline Hypericum perforatum $(\mathrm{H})(18)$ & Hypericaceae & 23.9 & $2003-0634$ \\
\hline Incarvillea delavayi $(\mathrm{H})(26)$ & Bignoniaceae & 31.8. & $2011-1215$ \\
\hline Lonicera tatarica & Caprifoliaceae & & $1997-0172$ \\
\hline Monarda didyma $(H)(5)$ & Lamiaceae & 30.9 & $1993-0230$ \\
\hline Mycelis muralis $(H)$ & Asteraceae & 30.9 & $2010-1327$ \\
\hline Pisum sativum $(\mathrm{H})$ & Fabaceae & 23.9 & $2005-0571$ \\
\hline Pisum sativum $(H)(27)$ & Fabaceae & 8.9 & $2012-1008$ \\
\hline Pisum sativum $(\mathrm{H})$ & Fabaceae & 23.9. & $2013-0762$ \\
\hline Pisum sativum $(\mathrm{H})$ & Fabaceae & 23.9 & $2010-0650$ \\
\hline Plantago lanceolata (H) (Amp) (20) & Plantaginaceae & 20.8 & $2006-0888$ \\
\hline Plantago major subsp. major (H) (Amp) (33) & Plantaginaceae & 10.9 & $2006-0889$ \\
\hline Plantago major subsp. major $(H)(2)$ & Plantaginaceae & 20.8 & $2008-1028$ \\
\hline Pulmonaria montana & Boraginaceae & & $2006-0654$ \\
\hline Quercus macrocarpa (H) (15) & Fagaceae & 30.9 & $1997-0448$ \\
\hline Quercus macrocarpa $(\mathrm{H})$ & Fagaceae & 23.9 & $1996-0015$ \\
\hline Quercus robur (H) (29) & Fagaceae & 31.8. & $00 X X-0256$ \\
\hline Quercus robur $(\mathrm{H})$ & Fagaceae & 30.9 & $00 X X-0035$ \\
\hline Rhamnus frangula (H) (Amp) (16) & Rhamnaceae & 30.9 & $1998-0201$ \\
\hline Rosa acicularis subsp. sayi $(H)(6)$ & Rosaceae & 3.8. & $1969-0232$ \\
\hline Rosa amblyotis & Rosaceae & & $1969-0282$ \\
\hline Rosa amblyotis & Rosaceae & & $1993-0734$ \\
\hline Rosa amblyotis (H) (11) & Rosaceae & 30.9 & $1993-0721$ \\
\hline Rosa blanda & Rosaceae & & $1990-0064$ \\
\hline Rosa maximowicziana (H) (Amp) (17) & Rosaceae & 23.9. & $1998-0600$ \\
\hline Rosa mollis & Rosaceae & & 1994-0443 \\
\hline Rosa nutkana & Rosaceae & & $1992-0531$ \\
\hline Rosa villosa & Rosaceae & & $2012-0542$ \\
\hline Rosa virginiana $(\mathrm{H})$ & Rosaceae & 30.9 & $1983-0565$ \\
\hline Salix caprea $(H)(A m p)(30)$ & Salicaceae & 3.8. & 1987-0943 \\
\hline Salix caprea $(\mathrm{H})$ & Salicaceae & 23.9. & $2010-0939$ \\
\hline Salix caprea $(H)(A m p)$ & Salicaceae & 30.9 & $00 X X-0095$ \\
\hline
\end{tabular}




\section{Host plant a, b}

\begin{tabular}{|c|c|c|c|}
\hline Salix hastata $(H)(42)$ & Salicaceae & 20.8 & $1977-0871$ \\
\hline Salix repens subsp. rosmarinifolia $(H)$ & Salicaceae & 31.8. & $1980-1929$ \\
\hline Salvia tesquicola & Lamiaceae & & $2012-0751$ \\
\hline Sambucus racemosa $(\mathrm{H})$ & Adoxaceae & 23.9. & $2010-0946$ \\
\hline Sambucus racemosa var. melanocarpa (H) (Amp) (41) & Adoxaceae & 3.8. & $1987-1194$ \\
\hline Solidago canadensis (H) (Amp) (14) & Asteraceae & 30.9 . & $1995-0741$ \\
\hline Stachys palustris $(H)$ & Lamiaceae & 30.9 & $2010-1389$ \\
\hline Stachys sylvatica (H) (Amp) (21) & Lamiaceae & 8.9 & $2007-0700$ \\
\hline Succisa pratensis $(H)(A m p)(24)$ & Dipsacaceae & 30.9 . & $2010-1341$ \\
\hline Succisa pratensis $(H)(19)$ & Dipsacaceae & 10.9 & $2006-0844$ \\
\hline Symphytum officinale var. bohemicum (H) (Amp) (8) & Boraginaceae & 20.8 & $1991-0452$ \\
\hline Thalictrum aquilegiifolium & Ranunculaceae & & 2013-0911 \\
\hline Thalictrum aquilegiifolium (H) (Amp) (22) & Ranunculaceae & 23.9 & $2008-1011$ \\
\hline Thalictrum lucidum $(\mathrm{H})(32)$ & Ranunculaceae & 8.9 & $2006-0610$ \\
\hline Tragopogon capitatus & Asteraceae & & $2009-0893$ \\
\hline Triticum aestivum & Poaceae & & 2009-0564 \\
\hline Triticum boeticum & Poaceae & & $2015-0361$ \\
\hline Triticum compactum & Poaceae & & $2015-0363$ \\
\hline Veronica jacquinii (H) (34) & Plantaginaceae & 23.9. & $2012-0760$ \\
\hline Veronica longifolia var. longifolia $(\mathrm{H})$ & Plantaginaceae & 30.9 & $2010-1391$ \\
\hline Veronica spicata $(\mathrm{H})(23)$ & Plantaginaceae & 8.9. & $2010-0870$ \\
\hline Viburnum lantana $(\mathrm{H})(\mathrm{Amp})(4)$ & Adoxaceae & 30.9. & $00 X X-0153$ \\
\hline Vicia sylvatica $(H)(3)$ & Fabaceae & 8.9 & $1991-0137$ \\
\hline
\end{tabular}

A samples 1-42 for ITS sequencing. Accession numbers refer to the KBG database (Kotka) and are also used to identify isolates subjected to DNA isolation.

b Hyperparasitic fungus (Ampelomyces quisqualis) was observed from the sample with a microscope (Amp).

c Dates of collection (in the year 2015) for inclusion in herbarium $(\mathrm{H})$. 


\section{Sequencing}

PCR products were purified using the E.Z.N.A. Gel Extraction Kit (Omega Bio-Tek, Georgia, USA). The purified DNA products were sequenced by Macrogen Inc. (Amsterdam, The Netherlands). Five samples (isolates 4, 8, 9, 28, and 33) were cloned into pGEM-T Easy vector (Promega Corporation, Madison) using E. coli $5 \alpha$ cells and sequenced with universal M13FpUC primer. This approach was used, because direct sequencing did not produce good-quality sequences (data not shown). All ITS1 and ITS2 sequences were deposited in European Nucleotide Archive (ENA) GenBank (accessions LT794916-LT795001).

\section{Analysis of sequences}

All ITS sequences obtained in this study were examined with BioEdit (v. 7.2.5, http://www.mbio.ncsu. edu/BioEdit/bioedit.html) software (Ibis Biosciences,
Carlsbad, CA, USA), and all ambiguous regions within the sequences were manually removed. The sequences were processed with the program ITSx (Bengtsson-Palme et al. 2013) to remove conservative rDNA sequences (18S, 5.8S, and 28S) and separate the ITS1 and ITS2 sequences of each isolate. ITS1 and ITS2 of the same sample were joined together using a text editor, followed by comparison with the sequences deposited in GenBank by applying the BLASTn tool (Altschul et al. 1990). Sequences (ITS1 + ITS2) were aligned with AliView software (v. 1.18) (Larsson 2014), and a phylogenetic tree was built using MEGA software (v. 7) (Kumar et al. 2016). The maximum likelihood method with 1000 bootstrap replicates (Felsenstein 1985) was used to estimate branch support. The evolutionary distances were counted with Kimura's two-parameter model (Kimura 1980). The ITS sequences of a closely related fungus, accession no. KT970793 (Baturo-Ciesniewska et al. 2017) from order Helotiales, were used as an outgroup.

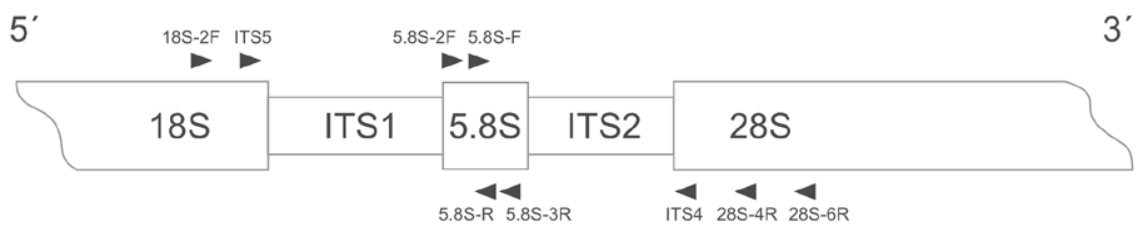

\begin{tabular}{ll}
\hline PRIMER & PRIMER SEQUENCE $\left(5^{\circ} \rightarrow 3^{\prime}\right)$ \\
\hline $\begin{array}{l}\text { Forward primers } \\
18 S-2 F \\
\text { ITS5 * }\end{array}$ & TGA ATG GCT AAG TGA GGC \\
$5.8 S-F$ & GGA AGT AAA AGT CGT AAC AAG G \\
$5.8 S-2 F$ & GTA ATG TGA ATT GCA GAA TTT \\
Reverse primers & TAAAAC TTT CAA CAA CGG ATC \\
$5.8 S-R$ & GCG CAA TGT GCG TTC AAA G \\
$5.8 S-3 R$ & GAA CAG GCA TGC CCC TMG \\
ITS4 * & TCC TCC GCT TAT TGA TAT GC \\
$28 S-4 R$ & CCA GAT TTC AAA TTT GAG CT \\
$28 S-6 R$ & AAG CAT CTT CTA CAAATT ACA \\
&
\end{tabular}

Fig. 2. Primers used for PCR and sequencing. The approximate binding sites of primers used for amplification of ITS1 and ITS2 regions are shown. 


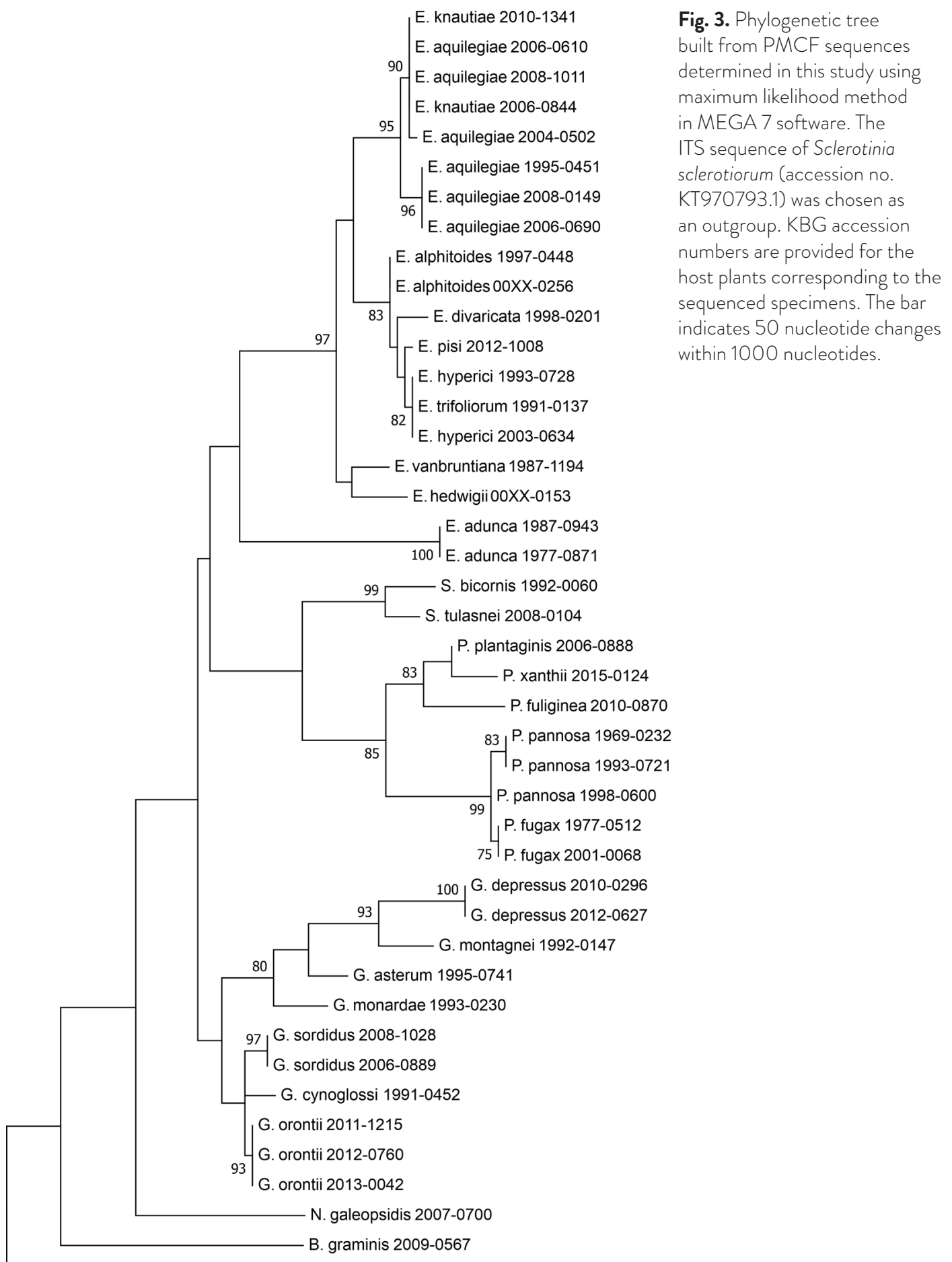

Fig. 3. Phylogenetic tree built from PMCF sequences determined in this study using maximum likelihood method en as KT ours are provided for the the the bar 50 nucleotide changes within 1000 nucleotides. 


\section{Results and discussion}

\author{
Morphological characteristics \\ and phylogenetic analysis
}

Altogether, 94 observations of powdery mildew were made on different host plants grown in KBG during the growth season of 2015 (Table 1), which represented 70 plant species from 24 families. Among the 70 herbarium samples collected, 14 species of PMCF were detected and identified. Their identification was based on morphological characteristics only (Supplementary Tables 1 and 2). A hyperparasitic fungus (Ampelomyces quisqualis sensu lato) was detected in 23 herbarium samples with a microscope (Table 1). Using the PMCF-specific primers, we were able to amplify rDNA from 37 of the 42 isolates (Table 2). The ITS regions were PCR-amplified from five PMCF samples in which multiple fungi were present and were cloned into pGEM-T Easy vector (Promega Corporation, Madison).

A phylogenetic tree of PMCF including six fungal genera was generated based on the identified sequences (Fig. 3) using BLASTn (Altschul et al. 1990). A few isolates lacked polymorphisms within their ITS sequences. They were assumed to be different species based on their minor morphological variations and previous knowledge of their known hosts as mentioned in the literature (Braun 1995; Braun \& Cook 2012).

\section{Taxonomy}

In this study, 28 species of PMCF from six genera were identified based on their morphological characteristics, ITS sequences, or both (Table 3). All PMCF determined in this study were previously known fungi in Finland. One of the identified fungi (Erysiphe hedwigii) lacks native hosts in Finland, yet it may thrive on ornamental host plants that are not native to Finland. The results of phylogenetic analyses and species determination based on morphological characteristics were largely consistent. The somewhat ambiguous species are discussed below.

\section{Erysiphe spp}

Eleven species of PMCF in the genus Erysiphe were identified based on knowledge of their morphological characteristics and analysis of ITS sequences (Table 3). A common feature in genus Erysiphe is production of a single conidium (Fig. 4a), whereas in the majority of other powdery mildew fungi the conidial-producing structures are characterized as catenescent (Fig. 4b). The appressoria in Erysiphe are typically lobed (Fig. 4c), and this feature helps to identify some samples. The fungal species $E$. hedwigii (isolate 00XX-0153) lacks a native host in Finland but may thrive on its ornamental hosts.

Erysiphe aquilegiae sensu lato infects plants within the family Ranunculaceae and is found throughout most of the world (Braun \& Cook 2012). There are two known variants of the fungus: var. $a q-$ uilegiae, which infects Thalictrum and Clematis, and var. ranunculi, which infects Aconitum, Clematis, Delphinium, and Thalictrum (Braun \& Cook 2012). In this study, the morphological characteristics were not sufficient to distinguish between these variants on their hosts, but the two clusters of E. aquilegiae branches in the phylogenetic tree (Fig. 3) support the variants mentioned by Braun \& Cook (2012). In contrast, Cunnington et al. (2004) showed that the ITS sequences are not the best candidates for distinguishing between these variants. On the same phylogenetic tree, isolates Erysiphe knautiae 20060844 and 2010-1341 showed $100 \%$ identity with isolates 2006-0610 and 2008-1011 of E. aquilegiae and 95\% identity with E. aquilegiae isolate 2004-0502. Takamatsu et al. (2015) showed that E. knautiae belongs to the homogenous clade E. aquilegia, which includes at least 15 fungal species that are identical or differ very little among their rDNA sequences. It is assumed that E. knautiae does not infect Ranunculaceae hosts but does infect hosts belonging to family Dipsacaceae (Braun \& Cook 2012). In Finland, E. knautiae is known to infect Knautia arvensis and Succisa pratensis (Braun 1995). The unexpected morphology of the appressoria of E. knautiae isolated in this study showed that appressoria in the observed samples were opposite (Fig. 4c) rather than single lobed, although they were described as being single lobed by Braun \& Cook (2012).

Erysiphe hyperici (Wallr.) S. Blumer and E. trifoliorum (Wallr.) U. Braun were identified based on knowledge of their hosts (Braun 1995; Braun \& Cook 2012) and ITS sequences. The ITS sequences show 
Table 2. The samples identified based on their

ITS sequence identities, as compared with previously

described fungi using the BLASTn tool.

\section{Fungus}

Blumeria graminis f. sp. avenae

Erysiphe adunca

Erysiphe adunca

Erysiphe alphitoides

Erysiphe alphitoides

Erysiphe aquilegiae

Erysiphe aquilegiae

Erysiphe aquilegiae

Erysiphe aquilegiae

Erysiphe aquilegiae

Erysiphe aquilegiae

Erysiphe divaricata

Erysiphe hedwigii

Erysiphe hyperici

Erysiphe hyperici

Erysiphe knautiae

Erysiphe knautiae

Erysiphe pisi

Erysiphe trifoliorum

Erysiphe vanbruntiana

Golovinomyces asterum var. solidaginis

Golovinomyces cynoglossi

Golovinomyces depressus

Golovinomyces depressus

Golovinomyces monardae

Golovinomyces montagnei

Golovinomyces orontii

Golovinomyces orontii

Golovinomyces orontii

Golovinomyces sordidus

Golovinomyces sordidus

Neoërysiphe galeopsidis

Podosphaera fugax

Podosphaera fugax

Podosphaera fuliginea

Podosphaera pannosa

Podosphaera pannosa

Podosphaera pannosa

Podosphaera plantaginis

Podosphaera xanthii

Sawadaea bicornis

Sawadaea tulasnei
Host plant

Avena sativa

Salix caprea

Salix hastata

Quercus macrocarpa

Quercus robur

Delphinium indet.

Aconitum napellus

Thalictrum aquilegiifolium

Clematis recta

Thalictrum lucidum

Delphinium elatum

Rhamnus frangula

Viburnum lantana

Hypericum ascyron

Hypericum perforatum

Succisa pratensis

Succisa pratensis

Pisum sativum

Vicia sylvatica

Sambucus racemosa var. melanocarpa

Solidago canadensis

Symphytum officinale var. bohemicum

Arctium lappa

Echium maculatum

Monarda didyma

Centaurea phrygia

Incarvillea delavayi

Veronica jacquinii

Cucumis sativus

Plantago major subsp. major

Plantago major subsp. major

Stachys sylvatica

Geranium sanquineum

Geranium pratense

Veronica spicata

Rosa acicularis subsp. sayi

Rosa amblyotis

Rosa maximowicziana

Plantago lanceolata

Cucurbita pepo

Acer negundo

Acer tataricum subsp. ginnala
Isolate

2009-0567

1987-0943

1977-0871

1997-0448

00XX-0256

2008-0149

1995-0451

2008-1011

2004-0502

2006-0610

2006-0690

1998-0201

$00 X X-0153$

1993-0728

2003-0634

2006-0844

2010-1341

2012-1008

1991-0137

1987-1194

1995-0741

1991-0452

2010-0296

2012-0627

1993-0230

1992-0147

2011-1215

2012-0760

2013-0042

2008-1028

2006-0889

2007-0700

1977-0512

2001-0068

2010-0870

1969-0232

1993-0721

1998-0600

2006-0888

2015-0124

1992-0060

2008-0104

\section{Sample}

$37 a$

$30 \mathrm{a}$

$42 \mathrm{a}$

$15 \mathrm{a}$

$29 a$

$1 a$

$13 a$

$22 \mathrm{a}$

$31 a$

$32 \mathrm{a}$

$38 a$

$16 b$

$4 c$

$12 \mathrm{a}$

$18 a$

$19 a$

$19 a$

24d

$27 a$

$3 a$

$41 a$

$14 \mathrm{a}$

$8 c$

$25 a$

$28 c$

$5 a$

$10 \mathrm{a}$

26 e

$34 \mathrm{a}$

$35 a$

$2 \mathrm{a}$

$33 c$

$21 a$

$7 a$

$39 d$

$23 a$

$6 a$

$11 a$

$17 \mathrm{~b}$

$20 \mathrm{a}$

$36 a$

$9 c$

$40 \mathrm{a}$

a PCR amplified with primer set 18S-2F/28S-4R and sequenced with primers 5.8SF and 5.8SR

b PCR amplified with primer set 18S-2F/28S-4R and 28S-6R, and sequenced with primers 5.8SF and 5.8S-3R

c PCR amplified with primer set 18S-2F/28S-6R and sequenced with M13F-pUC primer

d PCR amplified with primer set 18S-2F/28S-4R and sequenced with primers 5.8SF and 5.8S-3R

e PCR amplified with primer set $18 \mathrm{~S}-2 \mathrm{~F} / 28 \mathrm{~S}-4 \mathrm{R}$ and sequenced with primers $5.8 \mathrm{~S}-2 \mathrm{~F}$ and $5.8 \mathrm{~S}-3 \mathrm{R}$ 
Accession (ENA)

ITS1

LT794935

LT794916

LT794917

LT794918

LT794919

LT794922

LT794920

LT794923

LT794921

LT794924

LT794925

LT794926

LT794934

LT794928

LT794927

LT794929

LT794930

LT794931

LT794932

LT794933

LT794936

LT794937

LT794938

LT794939

LT794940

LT794941

LT794943

LT794944

LT794942

LT794945

LT794946

LT794949

LT794951

LT794950

LT794952

LT794953

LT794954

LT794955

LT794956

LT794957

LT794947

LT794948
ITS2

LT794977

LT794958

LT794959

LT794960

LT794961

LT794964

LT794962

LT794965

LT794963

LT794966

LT794967

LT794968

LT794976

LT794970

LT794969

LT794971

LT794972

LT794973

LT794974

LT794975

LT794978

LT794979

LT794980

LT794981

LT794982

LT794983

LT794985

LT794986

LT794984

LT794987

LT794988

LT794991

LT794993

LT794992

LT794994

LT794995

LT794996

LT794997

LT794998

LT794999

LT794989

LT794990
Length (bp)

324

396

396

396

396

392

392

392

392

392

392

400

395

398

398

392

392

398

398

395

349

345

345

345

353

350

346

346

346

346

346

344

318

316

316

317

317

317

316

316

313

303
BLASTn (ITS1 + ITS2)

Identity (\%)

Accession (ENA)

100

100

100

100

100

99

99

100

99

100

100

99

100

100

100

100

100

100

100

99

100

100

100

100

100

99

100

100

100

100

100

99

98

98

98

98

98

100

100

100

99

99
AJ313140.1

LC028970.1

LC028970.1

AB292705.1

AB292705.1

AY452802.1

AB921982.1

LC010016.1

LC010016.1

LC010016.1

LC009956.1

AF298539.1

LC010027.1

LC010027.1

LC010042.1

LC010042.1

LC009890.1

FJ378884.1

LC009909.1

KC513763.1

AB077684.1

AB077675.1

AB077675.1

LC076842.1

AB077656.1

AB769464.1

AB769464.1

AB769464.1

AB077658.1

AB077658.1

KX231842.1

AB525922.1

AB525922.1

AB046986.1

AB525937.1

AB525937.1

KX842352.1

JX442063.1

KX369541.1

AB193380.1

AB193385.1
AY452802.1 
Table 3. Summary of 28 PMCF species identified in the host plant samples collected from KBG and their identification methods. The accession numbers refer to the origin of the samples in the database of the KBG (https://kotka.luomus.fi).

\section{Fungus}

Blumeria graminis (DC.) Speer

Erysiphe adunca (Wallr.) Fr.

\section{Host plant (accession)}

Identification

Morphology ITS

Avena sativa (2009-0567) $\times$ ×

Hordeum vulgare (2009-0568)

Salix caprea (1987-0943)

Salix hastata (1977-0871)

Salix caprea (00XX-0095)

S. repens subsp. rosmarinifolia (1980-1929)

Salix caprea (2010-0939)

Erysiphe alphitoides (Griffon \& Maubl.) U.

Braun \& S. Takam.

Quercus macrocarpa (1997-0448)

Quercus robur (O0XX-0256)

Quercus robur (O0XX-0035)

Erysiphe aquilegiae DC.

Aconitum napellus (1995-0451)

$\times$

$\times$

$\times$

$\times$

$\times$

$\times$

$\times$

$\times \quad x$

$\times$

$\times \quad \times$

Clematis recta (2004-0502)

Delphinium elatum (2006-0690)

Delphinium indet. (2008-0149)

Thalictrum aquilegiifolium (2008-1011)

Thalictrum lucidum (2006-0610)

Delphinium elatum (1995-0091)

Erysiphe divaricata (Wallr.) Schltdl.

Rhamnus frangula (1998-0201)

Euonymus europaeus (1992-0099)

Erysiphe hedwigii (Lév.) U. Braun \& S. Takam. Viburnum lantana (O0XX-0153)

Erysiphe hyperici (Wallr.) S. Blumer

Hypericum ascyron (1993-0728)

Hypericum perforatum (2003-0634)

Erysiphe knautiae Duby

Succisa pratensis (2006-0844)

Succisa pratensis (2010-1341)

Erysiphe pisi DC.

Pisum sativum (2012-1008)
Erysiphe euonymi DC.
Erysiphe trifoliorum (Wallr.) U. Braun

Erysiphe vanbruntiana (W.R. Gerard)

U. Braun \& S. Takam.

Vicia sylvatica (1991-0137)

Sambucus racemosa (2010-0946)

S. racemosa var. melanocarpa (1987-1194)

Solidago canadensis (1995-0741)

Hieracium umbellatum (2010-1360)

Golovinomyces asterum var. solidaginis

V.P. Heluta

$\times \quad \times$

$\times$

$\times$

$\times$

$\times$

Golovinomyces cynoglossi (Wallr.) V.P. Heluta S. officinale var. bohemicum (1991-0452) 


\section{Fungus}

Host plant (accession)

Identification

Morphology ITS

\begin{tabular}{lll}
\hline Golovinomyces depressus (Wallr.) V.P. Heluta & Arctium lappa (2010-0296) & $\times$ \\
\cline { 2 - 3 } $\begin{array}{ll}\text { Golovinomyces monardae (G.S. Nagy) M. } \\
\text { Scholler }\end{array}$ & Monarda didyma (1993-0230) & $\times$ \\
\hline Golovinomyces montagnei U. Braun & Centaurea phrygia (1992-0147) & $\times$ \\
\hline Golovinomyces orontii (Castagne) V.P. Heluta & Cucumis sativus (2013-0042) & $\times$ \\
\cline { 2 - 3 } & Incarvillea delavayi (2011-1215) & $\times$ \\
\cline { 2 - 3 } & Veronica jacquinii (2012-0760) & $\times$ \\
\hline Golovinomyces sordidus (L. Junell) V.P. Heluta & Plantago major subsp. major (2006-0889) & $\times$ \\
\cline { 2 - 3 } & Plantago major subsp. major (2008-1028) & $\times$ \\
\hline Neoërysiphe galeopsidis (DC.) U. Braun & Stachys sylvatica (2007-0700) & $\times$ \\
\hline Podosphaera fugax (Penz. \& Sacc.) & Geranium pratense (2001-0068) & $\times$ \\
\cline { 2 - 3 } U. Braun \& S. Takam. & Geranium sanguineum (1977-0512) & $\times$ \\
\hline Podosphaera fuliginea (Schltdl.) & Veronica spicata (2010-0870) & $\times$ \\
U. Braun \& S. Takam. & & $\times$ \\
\hline Podosphaera pannosa (Wallr.) de Bary & Rosa acicularis subsp. sayi (1969-0232) & $\times$ \\
\cline { 2 - 3 } & Rosa amblyotis (1993-0721) & $\times$ \\
\cline { 2 - 3 } & Rosa maximowicziana (1998-0600) & $\times$ \\
\cline { 2 - 3 } & Rosa virginiana (1983-0565) & $\times$ \\
\hline Podosphaera plantaginis (Castagne) & Plantago lanceolata (2006-0888) & $\times$ \\
U. Braun \& S. Takam. & & $\times$ \\
\hline
\end{tabular}

Podosphaera xanthii (Castagne)

Cucurbita pepo (2015-0124)

U. Braun \& Shishkoff

Sawadaea bicornis (Wallr.) Homma
Acer negundo (1992-0060)

Sawadaea tulasnei (Fuckel) Homma

\begin{tabular}{ll} 
Acer platanoides (00XX-0004) & $\times$ \\
\hline Acer platanoides (00XX-0166) & $\times$ \\
\hline Acer tataricum subsp. ginnala (2008-0104) & $\times$ \\
\hline Acer tataricum subsp. tataricum & $\times$ \\
$(2005-0017)$ &
\end{tabular}

Acer tataricum subsp. tataricum (2008-0161) 
$100 \%$ similarity among all three isolates (1991-0137 on Vicia sylvatica, 1993-0728 on Hypericum ascyron, and 2003-0634 on H. perforatum) although they were collected from hosts belonging to different plant families. The morphological characteristics were not sufficient to distinguish between species. E. hyperici is a common fungus of Hypericum species in Asia, the Caucasus, Europe, and North America (Braun \& Cook 2012). There are also a few native hosts ( $H y$ pericum spp.) in Finland (Braun 1995; Mäkinen 1965; Rauhala 1957). In contrast, E. trifoliorum is known to infect only Fabacean hosts in Africa, North America, the Caucasus, and Europe. It has spread to South America, Australia, and New Zealand as well (Braun \& Cook 2012). The native hosts in Finland for E. trifoliorum are Succisa pratensis and several Lathyrus and Trifolium species (Ahti 1967; Braun 1995; Mäkinen 1965; Rauhala 1957). Also, genus Caragana is among the host plants in Finland (Braun 1995; Rauhala 1957), but Erysiphe palczewskii (Jacz.) U. Braun \& S. Takam. is known to have displaced E. trifoliorum on Caragana host plants in Finland (Huhtinen et al. 2001). Takamatsu et al. (2015) presented the clade E. trifoliorum sensu lato, which includes both E. hyperici and E. trifoliorum.

GOLOVINOMYCES SPP. Eight species of PMCF were identified as Golovinomyces with the methods previously mentioned (Table 3). A common morphological feature for the fungi in this genus is catenescent conidial formation (Fig. 4b). Similar to the Erysiphe species, all of the identified Golovinomyces species had been previously identified in Finland (Salo et al. 2019). The species Golovinomyces monardae (G.S. Nagy) M. Scholler (= G. biocellatus Ehrenb.) does not have any native Monarda plants as its host in Finland, but it is found on non-native ornamental hosts and on several native or non-native Lamiaceae hosts. In earlier publications, it is usually referred to as G. biocellatus, but Scholler et al. (2016) showed in their study that $G$. monardae can be excluded from the wider G. biocellatus complex and is classified as its own species.

Golovinomyces depressus (Wallr.) V.P. Heluta was identified in two species (Arctium lappa 20100296 and Echium maculatum 2012-0627) based on ITS sequencing. This may indicate that Echium maculatum is a new host for the fungus G. depressus, although the morphological characteristics were not enough to confirm this. In addition, pathogenicity was not tested to provide further proof. It is currently understood that G. depressus infects Arctium and Centaurea species in Asia, the Caucasus, Europe, and North America (Braun \& Cook 2012), but not Boraginaceae plants such as Echium spp. In Finland, the known $G$. depressus observations are from Arctium, Centaurea, and Onopordum acting as host plants (Braun 1995; Rauhala 1957).

\section{BLUMERIA, NEOËRYSIPHE, PODOSPHAERA, AND} SAWADAEA SPP. One Blumeria sp., one Neoërysiphe sp., five Podosphaera spp., and two Sawadaea spp. were identified (Table 3). Among them, Podosphaera and Sawadaea are the only species that have fibrosin bodies (Fig. 4e) inside their conidia, which can be used as a supportive feature for identification based on morphological characteristics. If teleomorphs are present, the species in genus Podosphaera are the only ones that have a single ascus inside each chasmothecia (Fig. 4d). Among all PMCF, Blumeria is the only genus that is capable of infecting monocot plants. Blumeria spp. share a common morphological feature of having a bulbous swelling (Fig. 4f) that develops from the hyphae and forms the base of the conidium. The species identified in these four genera are PMCF that had been previously identified in Finland (Salo et al. 2019).

Podosphaera fugax (Penz. \& Sacc.) U. Braun \& S. Takam. was identified in two Geranium samples $(G$. sanguineum 1977-0512 and G. pratense 2001-0068) based on morphological characteristics as well as ITS sequence similarity. Knowledge of the known hosts of P. fugax (Braun \& Cook 2012) was also used for species determination. Among ITS sequences from the fungus Podosphaera pannosa (Wallr.) de Bary isolated from three Rosa spp. (1969-0232, 1993-0721, and 1998-0600) there were differences at only a few nucleotides. All three $P$. pannosa sequences were 317 nucleotides in length but showed minor nucleotide polymorphisms among the analyzed isolates (19690232, 1993-0721, and 1998-0600). P. pannosa was also identified on a Rosa virginiana (1983-0565) herbarium sample based on morphological characteristics. P. fugax is known to infect Geranium species in Africa, Asia, the Caucasus, Europe, and North America, and it has been introduced into New Zealand (Braun \& Cook 2012). P. fugax is also a common fungus on Geranium spp. in the wild in Finland (Ahti 1967; Braun 


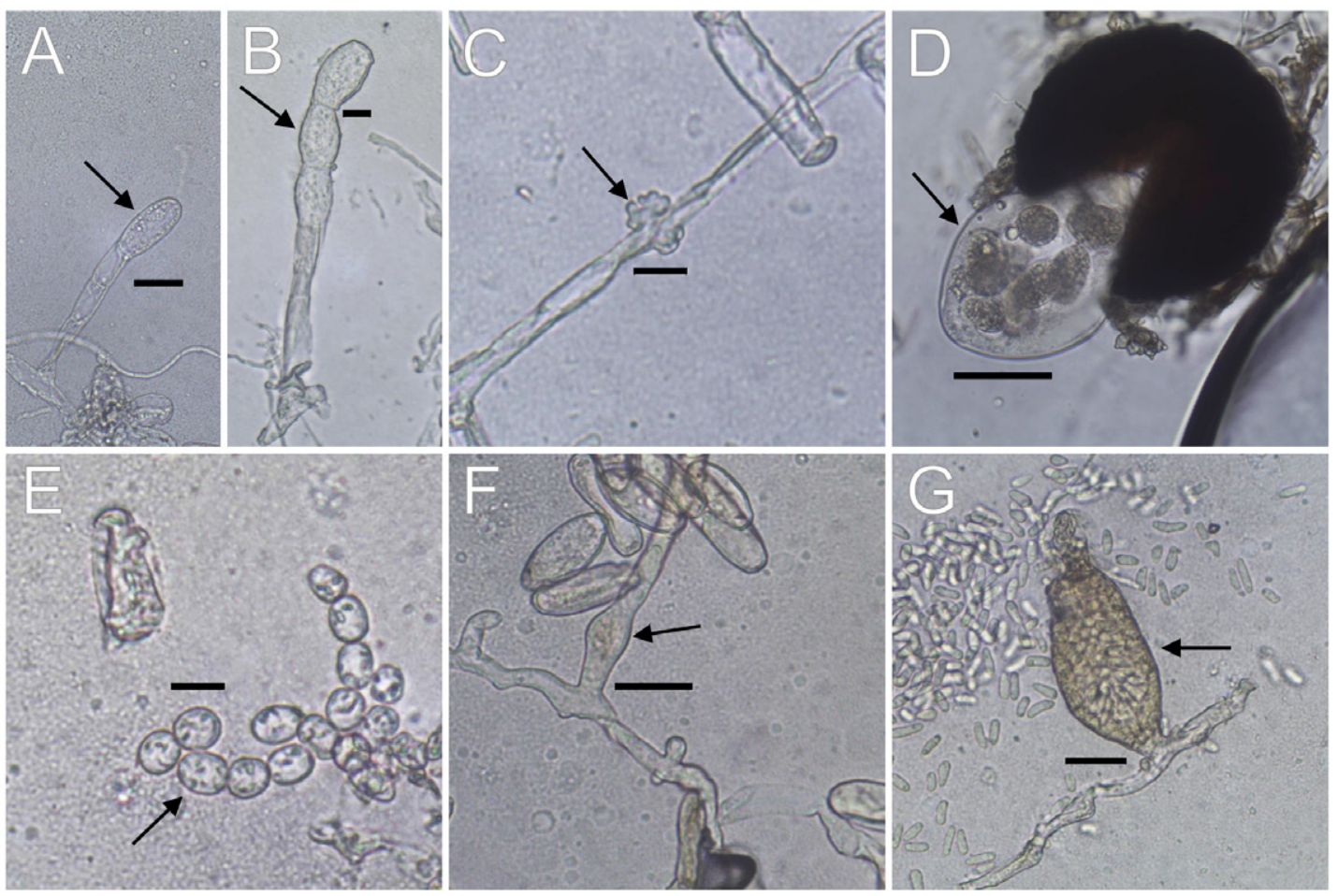

Fig. 4. Morphologically important characteristics of powdery mildew fungi. (a-g) The following are examples of structures observed among the 14 morphologically identifiable species of PMCF (host plant accession numbers are included): (a) a single conidiospore (1991-0137), bar = $20 \mu \mathrm{m}$; (b) a catenescent conidial formation (1993-0230), bar = $10 \mu \mathrm{m}$; (c) a lobed appressorium (2006-0844), bar = $10 \mu \mathrm{m}$; (d) a chasmothecium and single ascus containing multiple ascospores (1983-0565), bar = $40 \mu \mathrm{m}$; (e) microconidia and fibrosin bodies inside them (2005-0017), bar = $10 \mu \mathrm{m}$; (f) a bulbous swelling (2009-0568), bar = $20 \mu \mathrm{m}$; and (g) a hyperparasite of powdery mildew (1995-0741), bar $=20 \mu \mathrm{m}$.

1995; Rauhala 1957). Takamatsu et al. (2010) placed both $P$. fugax and $P$. pannosa in the same Rosoideae group, as they seem to be evolutionarily closely related. P. pannosa has spread all over the world (Braun \& Cook 2012) and has been found on several Rosa spp. in Finland (Braun 1995; Mäkinen 1965; Rauhala 1957).

Podosphaera plantaginis (Castagne) U. Braun \& S. Takam. was identified from one plant of Plantago lanceolata (isolate 2006-0888) based on ITS sequence similarity. P. plantaginis is known to infect plants in Asia, the Caucasus, Europe, and North America (Braun \& Cook 2012). The common host for the fungus in Finland is Plantago lanceolata (Braun 1995; Rauhala 1957; Jousimo et al. 2014). Ampelomyces quisqualis Ces. is a well-studied hyperparasitic fungus that infects P. lanceolata (Tollenaere et al. 2014) in Finland.
It grows inside the mycelial structures of the powdery mildew fungus (Fig. 4g) (Kiss et al. 2004). The ITS sequence of A. quisqualis was obtained in this study while sequencing the isolate from Plantago major 2006-0889. The $A$. quisqualis ITS sequence was deposited in ENA GenBank (ITS1: LT795000; ITS2: LT795001).

Taken together, the results of this study show that an area of six hectares can support a wide range of fungal populations, such as PMCF, when compatible host plants are present. Furthermore, the hyperparasitic fungus $A$. quisqualis sensu lato was commonly present based on microscope analysis. The 28 PMCF determined in this study are likely to represent just a subset of PMCF in Finland. However, they were isolated by one of the few surveys carried out in a botanical garden using morphological characteristics 
and molecular methods for identification. The higher number of PMCF identified with ITS sequences relative to morphological characteristics is supported by a previous study carried out elsewhere (Cunnington et al. 2003). The results of this study also show that botanical gardens maintain not only the genetic diversity of plants but also their pathogens, such as hyperparasitic fungi, which may help to regulate the impact of plants and microbes in gardens.

\section{Acknowledgments}

We thank Dr. Marko Hyvärinen and the personnel of Kumpula Botanic Garden for providing advice and space for the study. Financial support from Maiju ja Yrjö Rikala Foundation is gratefully acknowledged.

\section{References}

Ahti, T. 1967: Micromycetes (Peronosporaceae, Erysiphales and Uredinales) new to the province of Kuusamo, N. E. Finland. Karstenia 8: 5-8. https://doi.org/10.29203/ka.1968.47

Altschul, S.F., Gish, W., Millew, W., Myers, E.W. \& Lipman, D.J. 1990: Basic local alignment search tool. Journal of Molecular Biology 215: 403-410. https://doi.org/10.1016/S0022-2836(05)80360-2

Baturo-Ciesniewska, A., Groves, C.L., Albrecht, K.A., Grau, C.R., Willis, D.K. \& Smith, D.L. 2017: Molecular identification of Sclerotinia trifoliorum and Sclerotia sclerotiorum isolates from the United States and Poland. Plant Disease 101: 192-199. https://doi.org/10.1094/PDIS-06-16-0896-RE

Bengtsson-Palme, J., Ryberg, M., Hartmann, M., et al. 2013: Improved software detection and extraction of ITS1 and ITS2 from ribosomal ITS sequences of fungi and other eukaryotes for analysis of environmental sequencing data.

Methods in Ecology and Evolution 4: 914-919.

https://doi.org/10.1111/2041-210X.12073
Benson, D.A., Cavanaugh, M., Clark, K., Karsch-Mizrachi, I., Lipman, D.J., Ostell, J. \& Sayers, E.W. 2013: GenBank.

Nucleic Acids Research 41: D36-D42.

https://doi.org/10.1093/nar/gks1195

Braun, U. 1995: The powdery mildews (Erysiphales) of Europe. Gustav Fischer Verlag: Jena.

Braun, U. \& Cook, R.T.A. 2012: Taxonomic manual of the Erysiphales (Powdery mildew), CBS Biodiversity series no. 11, CBS-KNAW Fungal Diversity Centre: Utrecht.

Corpet, F. 1988: Multiple sequence alignment with hierarchical clustering. Nucleic Acids Research 16: 10881-10890. https://doi.org/10.1093/nar/16.22.10881

Cunnington, J.H., Lawrie, A.C. \& Pascoe, I.G. 2004:

Unexpected ribosomal DNA internal transcribed spacer sequence variation within Erysiphe aquilegiae sensu lato.

Fungal Diversity 16: 1-10.

http://www.fungaldiversity.org/fdp/sfdp/16-1.pdf

Cunnington, J.H., Takamatsu, S., Lawrie, A.C. \& Pascoe, I.G. 2003: Molecular identification of anamorphic powdery mildews (Erysiphales). Australasian Plant Pathology 32: 421-428. https://doi.org/10.1071/AP03045

Felsenstein, J. 1985: Confidence limits on phylogenies: an approach using the bootstrap. Evolution 39: 783-791. https://doi.org/10.1111/j.1558-5646.1985.tb00420.x

Glawe, D.A. 2008: The Powdery Mildews: a review of the world's most familiar (Yet poorly known) plant pathogens. Annual Review of Phytopathology 46: 27-51. https://doi.org/10.1146/annurev.phyto.46.081407.104740

Heffer, V., Johnson, K.B., Powelson, M.L. \& Shishkoff, N. 2006: Identification of Powdery Mildew Fungi anno 2006. The Plant Health Instructor. https://doi.org/10.1094/PHI-I-2006-0706-01

Huhtinen, S., Alanko, P. \& Mäkinen, Y. 2001: The invasion history of Microsphaera palczewskii (Erysiphales) in Finland. Karstenia 41: 31-36. https://doi.org/10.29203/ka.2001.376

Jousimo, J., Tack, A.J.M., Ovaskainen, O., Mononen, T., Susi, H., Tollenaere, C. \& Laine, A.L. 2014: Ecological and evolutionary effects of fragmentation on infectious disease dynamics. Science 344: 1289-1293.

https://doi.org/10.1126/science.1253621

Kimura, M. 1980: A simple method for estimating evolutionary rates of base substitutions through comparative studies of nucleotide sequences. Journal of Molecular Evolution 16: 111-120. https://doi.org/10.1007/BF01731581 
Kiss, L., Russell, J.C., Szentiványi, O., Xu, X. \& Jeffries, P. 2004: Biology and biocontrol potential of Ampelomyces mycoparasites, natural antagonists of powdery mildew fungi. Biocontrol Science and Technology 14: 635-651. https://doi.org/10.1080/09583150410001683600

Korytnanskaya, V.G., Tkachenko, F.P., Tovstuha, N.I. \& Rusanov, V.A. 2010: Powdery mildew fungi (Erysiphales) of Botanical Garden of Odessa National Mechnikov University. Chornomors'k. bot. z. 6: 259-264. https://doi.org/10.14255/2308-9628/10.62/9

Kumar, S., Stecher, G. \& Tamura, K. 2016: MEGA 7: Molecular evolutionary genetics analysis version 7.0 for bigger datasets. Molecular Biology and Evolution 33: 1870-1874.

Larsson, A. 2014: AliView: a fast and lightweight alignment viewer and editor for large data sets. Bioinformatics 30: 3276-3278. https://doi.org/10.1093/molbev/msw054

Mieslerová, B., Kitner, M., Petřeková, V., Dvořáková, J., Sedlárová, M., Cook, R.T.A. \& Lebeda, A. 2020a:

Golovinomyces powdery mildews on Asteraceae in the Czech Republic. Plant Protection Science 56: 163-179. https://doi.org/10.17221/129/2019-PPS

Mieslerová, B., Sedlářová, M., Michutová, M., Petřeková, V., Cook, R. \& Lebeda, A. 2020b: Powdery mildews on trees and shrubs in botanical gardens, parks and urban green areas in the Czech Republic. Forests 11: 967.

https://doi.org/10.3390/f11090967

Mäkinen, Y. 1965: On Finnish micromycetes. 7.

Recent additions to the distribution of powdery mildews in Finland. Annales Botanici Fennici 2: 243-247.

https://www.jstor.org/stable/23724118

Rauhala, A. 1957: Mehltauspilzfunde aus Finnland mit berücksichtigung der bisherigen verbreitungsangaben. Karstenia 4: 14-26. https://doi.org/10.29203/ka.1958.33

Salo, P., Ahti, T. \& Salo, V. 2019: Erysiphales, in: Suomen Lajitietokeskus 2019: Lajiluettelo 2018. Suomen Lajitietokeskus, Luonnontieteellinen keskusmuseo, Helsingin yliopisto, Helsinki. http://urn.fi/URN:ISSN:2490-0907.

Accessed 8 June 2020

Schulman, L. \& Hällfors, M. 2011: Botanical garden profile: Kumpula botanic garden, Helsinki, Finland. Sibbaldia: The Journal of Botanic Garden Horticulture 9: 11-28. https://journals.rbge.org.uk/index.php/rbgesib/article/ view/119/109
Schoch, C.L., Seifert, K.A., Huhndorf, S., Robert, V., Spouge, J.L., Levesque, C.A., Chen, W. \& Fungal Barcoding Consortium 2012: Nuclear ribosomal internal transcribed spacer (ITS) region as a universal DNA barcode marker for Fungi. Proceedings of the National Academy of Sciences of the United States of America 109: 6241-6246. https://doi.org/10.1073/pnas.1117018109

Scholler, M., Schmidt, A., Siahaan, S.A.S., Takamatsu, S. \& Braun, U. 2016: A taxonomic and phylogenetic study of the Golovinomyces biocellatus complex (Erysiphales, Ascomycota) using asexual state morphology and rDNA sequence data. Mycological Progress 15: 1-13. https://doi.org/10.1007/s11557-016-1197-5

Takamatsu, S., Ito, H., Shiroya, Y., Kiss, L. \& Heluta, V. 2015: First comprehensive phylogenetic analysis of the genus Erysiphe (Erysiphales, Erysiphaceae) I. The Microsphaera lineage. Mycologia 107: 475-489. https://doi.org/10.3852/15-007

Takamatsu, S., Niinomi, S., Harada, M. \& Havrylenko, M. 2010: Molecular phylogenetic analyses reveal a close evolutionary relationship between Podosphaera (Erysiphales: Erysiphaceae) and its rosaceous hosts. Persoonia 24: 38-48. https://doi.org/10.3767/003158510X494596

Tollenaere, C., Pernechele, B., Mäkinen, H.S., Parratt, S.R., Németh, M.Z., Kovács, G.M., Kiss, L., Tack, A.J.M. \& Laine, A.L. 2014: A hyperparasite affects the population dynamics of a wild plant pathogen. Molecular Ecology 23: 5877-5878. https://doi.org/10.1111/mec.12908

Weltzien, H.C. 1978: Geographical distribution of powdery mildews. In: Spencer, D.M. (ed). The powdery mildews, 39-49. Academic Press, London.

White, T.J., Bruns, T.D., Lee, S. \& Taylor, J.W. 1990: Amplification and direct sequencing of fungal ribosomal RNA genes for phylogenetics. In: Innis, M.A., Gelfand, D.H., Sninsky, J.J. \& White, T.J. (eds). PCR protocols: A guide to methods and applications, 315-322. Academic Press, London.

\section{SUPPLEMENTARY TABLES}

Table S1. Fungal isolates observed at the anamorph stage with a focus on their mycelia and conidia.

Table S2. Fungal isolates observed at the teleomorph stage with a focus on chasmothecia, asci, and ascospores. 